\title{
Multivariate Analyse von qualitativen abhängigen Variablen mit verallgemeinerten linearen Modellen*
}

\author{
Gerhard Arminger \\ Universität Gesamthochschule Wuppertal, FB Wirtschaftswissenschaften \\ Gaußstraße 20, D-5600 Wuppertal
}

$\mathrm{Z}$ u s a m m e $\mathrm{n}$ a s $s$ u $\mathrm{n}$ : Die bisher verwendeten Modelle und Programme (ECTA, NONMET) zur Analyse von qualitativen abhängigen Variablen werden auf verallgemeinerte lineare Modelle zurückgeführt. Damit werden auch die Probleme der Einbeziehung von quantitativen unabhängigen Variablen sowie der Berechnung von Freiheitsgraden und der Schätzung von Parametern bei fehlenden Zellen auf einfache Weise gelöst.

„Das Gewebe dieser Welt ist aus Zufall und Notwendigkeit gebildet."

(Goethe 1796) meinerten linearen Modell (in Zukunft mit GLMAnsatz oder GL-Modell bezeichnet) folgende Probleme lösen lassen, die in den bisher verwendeten Ansätzen Schwierigkeiten bereiten:

- Die korrekte Behandlung fehlender Zellenwerte in Kontingenztabellen. Sowohl in ECTA als auch in NONMET lassen sich Haupt- und Interaktionseffekte nur schätzen, wenn alle Zellen besetzt sind. Es werden daher bei nicht besetzten Zellen künstlich kleine Besetzungen (z.B. 0.5 im dichotomen Fall) vorgegeben, die zu einer falschen Anzahl von Freiheitsgraden führen.

- Die Einbeziehung von quantitativen unabhängigen Variablen. (Diese Einbeziehung kann auch auf NONMET übertragen werden.)

- Für loglineare Modelle kann die geschätzte Kovarianzmatrix der Haupt- und Interaktionseffekte angegeben werden, so daß beliebige lineare Kontraste - z.B. Differenzen zwischen zwei Effekten - getestet werden können.

Zusätzlich wird gezeigt, daß sowohl die in ECTA als auch die in NONMET verwendeten Modelle spezielle GL-Modelle sind und sich alle mit diesen Ansätzen erzeugten Ergebnisse auch mit GLIM reproduzieren lassen. Mit diesem Ergebnis ist die zwischen Küchler (1978) und Langeheine (1979) geführte Kontroverse um NONMET versus ECTA im Hegelschem Sinne aufgehoben.

\section{Beispiel: Erwerbstätigkeit verheirateter Frauen}

\subsection{Darstellung und Interpretation eines logit Modells}

Im Rahmen des VASMA Projektes an der Universität Mannheim wird unter anderem die Ent- 
wicklung der Frauenerwerbstätigkeit im zeitlichen Verlauf untersucht. Dem Datenbestand von VASMA wurde Tabelle 1 entnommen, die die Erwerbstätigkeit verheirateter Frauen der Geburtsjahrgänge 1931-1941 im Mikrozensus 1971 angibt. Die Ehemänner sind nicht selbständig.

Zur inhaltlichen Diskussion um die Erwerbstätigkeit von Frauen verweisen wir auf die Arbeiten im Rahmen des VASMA Projektes (Müller 1981; Handl 1981; Willms 1981) sowie auf Mincer und Polachek (1974) und Cramer (1980).

Um das Datenmaterial übersichtlich zu halten, wurden die im Mikrozensus angeführten Ausprägungen der folgenden Variablen auf die hier angegebenen Ausprägungen reduziert.

X Einkommen des Mannes (Monatsdurchschnitt)

$\mathrm{X} 12000 \mathrm{DM}$

$\mathrm{X} 21500 \mathrm{DM}$

X3 $1000 \mathrm{DM}$

$\mathrm{X} 4700 \mathrm{DM}$

X5 $450 \mathrm{DM}$

A Schulbildung der Frau
A1 Nur Volksschule
A2 Mittlere Ausbildung
A3 Höhere Ausbildung

B Kinder
B1 Keine Kinder
B2 Kinder, die älter als 6 Jahre sind
B3 Kinder, die jünger als 6 Jahre sind

R Zahl der nicht erwerbstätigen Frauen in der Kombination XAB

$\mathrm{N}$ Zahl der Frauen insgesamt (Erwerbstätige und Nicht-Erwerbstätige) in der Kombination XAB.

(Erwerbstätigkeit ist dabei auf un/angelernte Arbeiterin und ausfuhrende Angestellte oder Beamtin beschränkt.)

Z Einkommen des Mannes als quantitative Variable aufgefaßt (in hundert DM skaliert).

Man beachte, daß die Kombinationen X5 A3 (geringes Einkommen des Mannes, höhere Ausbildung der Frau) und X4 A3 B2 überhaupt nicht auftreten. Diese Kombinationen müssen wir als strukturelle Nullen auffassen, da aus alge-
TABELLE 1: Erwerbstätigkeit verheirateter Frauen im Mikrozensus 1971

\begin{tabular}{|c|c|c|c|c|c|}
\hline $\mathbf{X}$ & $\mathbf{A}$ & B & $\mathbf{R}$ & $\mathbf{N}$ & $Z$ \\
\hline 5 & 1 & 1 & 0016 & 0032 & 04.5 \\
\hline 5 & 1 & 2 & 0052 & 0096 & 04.5 \\
\hline 5 & 1 & 3 & 0043 & 0057 & 04.5 \\
\hline 5 & 2 & 1 & 0005 & 0016 & 04.5 \\
\hline 5 & 2 & 2 & 0013 & 0035 & 04.5 \\
\hline 5 & 2 & 3 & 0017 & 0026 & 04.5 \\
\hline 4 & 1 & 1 & 0132 & 0383 & 07.0 \\
\hline 4 & 1 & 2 & 0640 & 1155 & 07.0 \\
\hline 4 & 1 & 3 & 0607 & 0793 & 07.0 \\
\hline 4 & 2 & 1 & 0047 & 0217 & 07.0 \\
\hline 4 & 2 & 2 & 0260 & 0461 & 07.0 \\
\hline 4 & 2 & 3 & 0265 & 0364 & 07.0 \\
\hline 4 & 3 & 1 & 0001 & 0003 & 07.0 \\
\hline 4 & 3 & 3 & 0000 & 0001 & 07.0 \\
\hline 3 & 1 & 1 & 0329 & 0845 & 10.0 \\
\hline 3 & 1 & 2 & 2925 & 4398 & 10.0 \\
\hline 3 & 1 & 3 & 2838 & 3359 & 10.0 \\
\hline 3 & 2 & 1 & 0242 & 0913 & 10.0 \\
\hline 3 & 2 & 2 & 1874 & 2926 & 10.0 \\
\hline 3 & 2 & 3 & 2384 & 2877 & 10.0 \\
\hline 3 & 3 & 1 & 0001 & 0013 & 10.0 \\
\hline 3 & 3 & 2 & 0009 & 0014 & 10.0 \\
\hline 3 & 3 & 3 & 0013 & 0015 & 10.0 \\
\hline 2 & 1 & 1 & 0100 & 0207 & 15.0 \\
\hline 2 & 1 & 2 & 0927 & 1246 & 15.0 \\
\hline 2 & 1 & 3 & 1022 & 1126 & 15.0 \\
\hline 2 & 2 & 1 & 0178 & 0617 & 15.0 \\
\hline 2 & 2 & 2 & 1581 & 2036 & 15.0 \\
\hline 2 & 2 & 3 & 2118 & 2420 & 15.0 \\
\hline 2 & 3 & 1 & 0010 & 0023 & 15.0 \\
\hline 2 & 3 & 2 & 0039 & 0051 & 15.0 \\
\hline 2 & 3 & 3 & 0095 & 0109 & 15.0 \\
\hline 1 & 1 & 1 & 0016 & 0032 & 20.0 \\
\hline 1 & 1 & 2 & 0143 & 0162 & 20.0 \\
\hline 1 & 1 & 3 & 0147 & 0153 & 20.0 \\
\hline 1 & 2 & 1 & 0106 & 0199 & 20.0 \\
\hline 1 & 2 & 2 & 0722 & 0820 & 20.0 \\
\hline 1 & 2 & 3 & 0908 & 0960 & 20.0 \\
\hline 1 & 3 & 1 & 0016 & 0023 & 20.0 \\
\hline 1 & 3 & 2 & 0094 & 0102 & 20.0 \\
\hline 1 & 3 & 3 & 0209 & 0217 & 20.0 \\
\hline
\end{tabular}

braischen Gründen keine Maximum Likelihood Lösung, die später allgemein angegeben wird, existiert. Die Kombination X4 A3 B3 tritt nur bei den Erwerbstätigen auf. $\mathrm{Da}$ die Stichprobe in dieser Kombination die Größe 1 hat, fassen wir die Null bei den Nicht-Erwerbstätigen als Stichproben-Null auf.

Wir wollen nun die Zahl der nicht erwerbstätigen Frauen erklären durch die qualitativen unabhängigen Variablen Ausbildung (A) und Kind (B) und durch die quantitative Variable Einkommen des Ehemanns $(Z)$. Wir verwenden 
zur Erklärung ein logit Modell mit der abhängigen Variablen

$\eta_{\mathrm{i}}=\ln \left(\mathrm{R}_{\mathrm{i}} /\left(\mathrm{N}_{\mathrm{i}}-\mathrm{R}_{\mathrm{i}}\right)\right)$ für jede Kombination $\mathrm{ABZ}$, $\mathrm{i}=1, \ldots 41$

und rechnen ein GLM, das analog zu einer gewichteten Kovarianzanalyse aufgebaut und interpretierbar ist.

Wir verwenden ein logit Modell, da es - wie wir später an diesem Beispiel zeigen werden - bei gleicher Parameterstruktur - wesentlich besser mit den beobachteten Werten übereinstimmt als ein lineares Modell mit Prozentsätzen.

$\eta_{\mathrm{i}}=\mathbf{R}_{\mathrm{i}} / \mathrm{N}_{\mathrm{i}}, \mathrm{i}=1, \ldots 41$

Ist das aus einem logit Modell geschätzte $\eta_{\mathrm{i}}$ berechnet, erhalten wir den prognostizierten Wert $\hat{\mathrm{R}}_{\mathrm{i}}$ aus der Umkehrtransformation

$\hat{\mathrm{R}}_{\mathrm{i}}=\mathrm{N}_{\mathrm{i}} \hat{\pi}_{\mathrm{i}}$ mit $\hat{\pi}_{\mathrm{i}}=\exp \left(\eta_{\mathrm{i}}\right) /\left(1+\exp \left(\hat{\eta}_{\mathrm{i}}\right)\right)$

Der Zusammenhang zwischen $\eta$ und $\pi$ wird durch folgendes Schaubild illustriert:

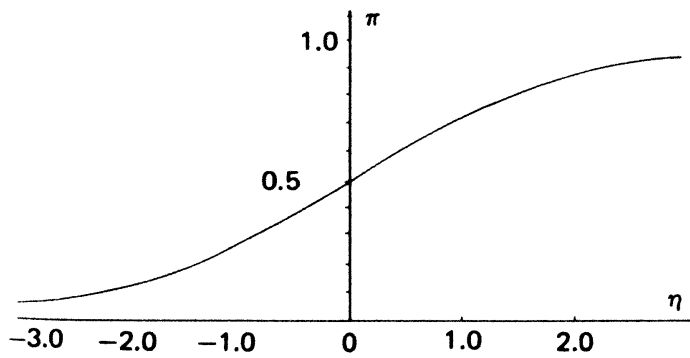

FIGUR 1: Graph der Funktion $\pi=\exp (n) /(1+$ $\exp (\eta))$

Auf Grund von Uberlegungen über die Stärke der einzelnen Variablen, die anschließend angegeben werden, haben wir folgendes Modell gewählt:

$\eta_{i j k}=\beta_{o}+\beta_{i}^{A} \delta_{i}^{A}+\beta_{j}^{B} \delta_{j}^{B}+\beta^{Z} Z_{k}+\beta_{i j}^{A B} \delta_{i}^{A} \delta_{j}^{B}+$ $\beta_{j}^{B Z} \delta_{j}^{B} Z_{k}$

$i=1,2,3 ; j=1,2,3 ; k=1, \ldots 5$

${ }_{0}^{6} \mathbf{A}, \delta_{j} \mathbf{B}$ sind Dummy Variable

$\delta_{i}^{A}=\left\{\begin{array}{l}1 \text { wenn Ausprägung } i \text { von } A \text { auftritt } \\ 0 \text { sonst und bei } i=1 \text { (Reparametrisie- }\end{array}\right.$ rungsbedingung)
$\delta_{\mathrm{j}}^{B}=\left\{\begin{array}{l}1 \text { wenn Ausprägung } \mathrm{j} \text { von } \mathrm{B} \text { auftritt } \\ 0 \text { sonst und bei } \mathrm{j}=1 \text { (Reparametrisie- }\end{array}\right.$ rungsbedingung)

$Z_{k}=$ quantitative Variable

$\beta_{0}=$ Regressionskonstante

$\beta_{\mathrm{i}}^{\mathrm{A}}, \beta_{\mathrm{j}}^{\mathrm{B}}, \beta_{\mathrm{ij}}^{\mathrm{AB}}=$ Haupt- und Interaktionseffekte der

$\beta^{Z}=$ Regressionskoeffizient von $Z$

$\beta_{j}^{\mathrm{BZ}}=$ Veränderung des Regressionskoeffizienten von $Z$ in den Ausprägungen $B 2$ und $\mathrm{B} 3$ gegenüber $\mathrm{B} 1$.

Die diesem Modell entsprechende GLIM Formulierung ist

$A+B+Z+A . B+B . Z$,

indem die unabhängigen Variablen angegeben werden, die zur Erklärung von $\eta_{\mathrm{i}}$ herangezogen werden.

Mit einem GLIM-Programm erhalten wir folgendes Ergebnis:

Der Wert DEVIANCE in GL-Modellen nimmt für loglineare bzw. logit-Modelle den Wert $\mathbf{G}^{2}$ (Bishop et al. 1975) an und ist - falls die $\mathrm{H}_{\mathrm{o}}$ : die geschätzten Werte $\hat{R}_{i}$ unterscheiden sich nur zufälig von den beobachteten Werten $R_{i}$ - asymptotisch $\chi^{2}$-verteilt mit DF Freiheitsgraden.

$\mathrm{n} \quad=$ Anzahl der Zellen $\mathrm{ABZ}$, also $\mathrm{n}=41$

$\mathrm{p}=$ Anzahl der geschätzten Parameter, also $\mathrm{p}=12$

$D F=n-p=29$

$G^{2}=\sum_{i=1}^{n}\left[R_{i} \ln \left(R_{i} / \hat{R}_{i}\right)+\left(N_{i}-R_{i}\right) \ln \left(\left(N_{i}-R_{i}\right) /\right.\right.$

$$
\left.\left.\left(\mathrm{N}_{\mathrm{i}}-\hat{\mathrm{R}}_{\mathrm{i}}\right)\right)\right]
$$

DEVIANCE $=71.73>\chi_{29 ; 0.95}^{2}=42.6$, d.h. das geschätzte Modell weicht noch von den Daten signifikant ab. Da DEVIANCE wesentlich von der Größe der Stichprobe abhängt, behalten wir trotzdem zunächst dieses Modell bei.

Die geschätzten Werte der einzelnen Parameter (ESTIMATE), ihre Standardabweichungen (S.E. = standard error) und Bezeichnungen sind angegeben, die untere Dreiecksmatrix enthält die Standardabweichungen aller Paare von Differenzen. 
TABELLE 2 Schätzungen der Parameter, ihrer Standardabweichungen und der Standardabweichungen der

Differenzen im Modell A + B + Z + A.B + B. Z

\begin{tabular}{rlll}
\hline & $\begin{array}{c}\text { Deviance } \\
71.73\end{array}$ & $\begin{array}{l}\text { DF } \\
29\end{array}$ & \\
\hline & ESTIMATE & S.E. & PARAMETER \\
\hline 1 & -1.208 & .1179 & $<$ GM \\
2 & -.6325 & $.7753 \mathrm{E}-01$ & A $(2)$ \\
3 & -.1923 & .2699 & A (3) \\
4 & .5938 & .1361 & B (2) \\
5 & 1.640 & .1500 & B (3) \\
6 & $.7784 \mathrm{E}-01$ & $.1040 \mathrm{E}-01$ & Z \\
7 & .6004 & $.8706 \mathrm{E}-01$ & A $(2) . \mathrm{B}(2)$ \\
8 & .4463 & $.9443 \mathrm{E}-01$ & $\mathrm{~A}(2) . \mathrm{B}(3)$ \\
9 & .3964 & .3514 & A (3).B (2) \\
10 & .1457 & .3482 & A (3).B (3) \\
11 & $.4724 \mathrm{E}-01$ & $.1211 \mathrm{E}-01$ & B (2).Z \\
12 & $.4569 \mathrm{E}-01$ & $.1334 \mathrm{E}-01$ & B (3).Z \\
\hline
\end{tabular}

S.E. of Differences

0.

$\begin{array}{llllll}.1416 & 0 . & & & & \\ .2836 & .2650 & 0 . & & & \\ .2454 & .1562 & .3126 & 0 . & & \\ .2534 & .1684 & .3189 & .1150 & 0 . & \\ .1273 & 8.1639 \mathrm{E}-02 & .2723 & .1283 & .1429 & 0 . \\ .1461 & .1600 & .2984 & .1619 & .1738 & 8.4515 \mathrm{E}-02 \\ .1506 & .1642 & .3007 & .1661 & .1787 & 9.2086 \mathrm{E}-02 \\ .3790 & .3716 & .5848 & .3631 & .3737 & .3498 \\ .3761 & .3686 & .5829 & .3653 & .3614 & .3466 \\ .1089 & 7.4920 \mathrm{E}-02 & .2680 & .1471 & .1576 & 2.1709 \mathrm{E}-02 \\ .1090 & 7.5129 \mathrm{E}-02 & .2681 & .1446 & .1621 & 2.2420 \mathrm{E}-02 \\ 1 & 2 & 3 & 4 & 5 & 6\end{array}$

70.

$8 \quad 6.6882 \mathrm{E}-02 \quad 0$.

$\begin{array}{lll}9 & .3468 & .3518\end{array}$

$10 \quad .3446 \quad .3420$

$11 \quad 9.1668 \mathrm{E}-02$

$12 \quad 9.1119 \mathrm{E}-02$

$9.8022 \mathrm{E}-02$

0. $9.9424 \mathrm{E}-02$

.3146

.3540

.3533

9
0.

$.3501 \quad 0$.

$.3515 \quad 1.0401 \mathrm{E}-020$.

$\begin{array}{lll}10 & 11 & 12\end{array}$
Auf Grund der Setzungen der Dummy Variablen $\delta_{i}^{A}, \delta_{j}^{B}$ werden die Haupt- und Interaktionseffekte der jeweils ersten Ausprägungen gleich $\mathbf{0}$ gesetzt. Die erste Kategorie einer Variablen dient also jeweils als Bezugskategorie.

Die Interpretation ist daher folgende:

GM ist die Regressionskonstante. Sie gibt - nach Transformation - die Wahrscheinlichkeit an, daß eine Frau mit Volksschulbildung (A1) ohne Kind (B1) und keinem Einkommen des Ehemannes $(Z=0)$ nicht erwerbstätig ist. $\hat{\pi}=\exp (\mathrm{GM}) /(1+\exp (\mathrm{GM}))=\exp (-1.208) /$ $(1+\exp (-1.208))=0.229$

Im Vergleich zu A1 - nur Volksschulbildung wird die Wahrscheinlichkeit, nicht erwerbstätig zu sein, verringert, wenn die Frau mittlere Ausbildung $(A(2)=-0.6325)$ oder höhere Ausbildung $(\mathrm{A}(3)=-0.1923)$ besitzt. Allerdings ist $A(3)$ nicht signifikant von 0 verschieden. (Die Koeffizienten $\beta_{i}^{A}, \beta_{j}^{B}, \beta^{Z}$ usw. werden jeweils mit den Variablennamen in GLIM bezeichnet.)

Man kann nämlich davon ausgehen, daß die Parameter in guter Näherung normalverteilt sind mit 
der angegebenen Standardabweichung. Das 95\% Konfidenzintervall um -0.1923 ist daher (S.E.= 0.2699 ):

$\mathrm{P}(-0.1923-1.96 \cdot 0.2699 \leqslant \mathrm{~A}(3) \leqslant-0.1923$ $+1.96 \cdot 0.2699) \geqslant 0.95$

Da der Wert 0 in diesem Intervall enthalten ist, läßt sich die $\mathrm{H}_{0}: A(3)=0$ gegen $\mathrm{H}_{1}: A(3)=0$ nicht mit Irrtumswahrscheinlichkeit $\alpha=0.05$ verwerfen.

Die Variable B beeinflußt die Erwerbstätigkeit wie folgt:

Kinder über $6(\mathrm{~B}(2)=.5938)$ und Kinder unter $6(B(3)=1.640)$ erhöhen die Wahrscheinlichkeit, nicht erwerbstätig zu sein, beträchtlich.

Als Beispiel berechnen wir die Wahrscheinlichkeit der Nicht-Erwerbstätigkeit, wenn eine Frau Volksschulausbildung hat, Kinder unter 6 im Haus sind und der Ehemann ein Einkommen von $Z=10=1000 \mathrm{DM}$ hat.

$$
\begin{aligned}
\eta & =\mathrm{GM}+\mathrm{A}(1)+\mathrm{B}(3)+\hat{\beta}^{\mathrm{z}} \cdot \mathrm{Z} \\
= & -1.208+0+1.640+0.07784 \cdot 10= \\
& 1.2104 \\
\hat{\pi} & =\exp (1.2104) /(1+\exp (1.2104))=0.77
\end{aligned}
$$

Der Parameter $\hat{\beta} Z=0.07784$ gibt den Einfluß des Einkommens an. Der Regressionskoeffizient ist positiv, d.h. je höher das Einkommen, um so höher ist die Wahrscheinlichkeit der Nichterwerbstätigkeit. Der Effekt von $\mathrm{Z}$ ist ebenfalls beträchtlich, da ja jeweils das Einkommen mit diesem Koeffizienten multipliziert werden muß.

Die Interaktionen erhöhen jeweils die Wahrscheinlichkeit der Nichterwerbstätigkeit. Die Kombination A2B2, also mittlere Ausbildung und Kinder über 6 Jahre, erhöht die Wahrscheinlichkeit, nicht erwerbstätig zu sein, im Ausmaß von $A(2) \cdot B(2)=0.6004$. Dies bedeutet, daB der negative Effekt der mittleren Ausbildung auf Nichterwerbstätigkeit zurückgenommen wird, sobald Kinder in der Familie sind.

Die Interaktionen $\mathrm{B}(2) \cdot \mathrm{Z}=0.04724$ und $B(3) \cdot Z=0.04569$ zeigen auf, da $B$ der Regressionskoeffizient von $Z$ erhöht wird, wenn die Familie Kinder hat. Der positive Einfluß des Einkommens auf Nichterwerbstätigkeit wird also noch verstärkt.
Wir testen noch, ob sich $B(2) \cdot Z$ und $B(3) \cdot Z$ voneinander unterscheiden:

$H_{0}: \Delta=B(2) \cdot Z-B(3) \cdot Z$ gegen $H_{1}: \Delta \neq 0$

Da $\Delta=0.04724-0.04569=0.00165$ und die Standardabweichung der Differenz zwischen Parameter 11 und $12=0.00104$ beträgt, kann $H_{0}$ nicht mit $\alpha=0.05$ abgelehnt werden.

Insgesamt ist die Interpretation dieser Werte völlig analog zur Kovarianzanalyse zu bewerkstelligen. Ungewohnt ist zunächst nur die logit Transformation; sie verhindert jedoch, daß z.B. bei sehr hohem Einkommen Schätzwerte für die Wahrscheinlichkeit der Nichterwerbstätigkeit auftreten können, die größer als 1 sind.

Einen weiteren Begriff von der Güte der Anpassung erhalten wir, wenn wir die beobachteten Werte von $\mathbf{R}$ (observed) mit den unter dem Modell erwarteten Werten (fitted) von $\hat{R}$ vergleichen. Die Residuen sind standardisiert und - wenn das Modell zutrifft - annähernd $\mathrm{N}(0,1)$ verteilt. Als Faustregel bedeutet dies, daß Werte, die dem Betrag nach größer als 2 sind, nur ca. 5\% aller Residuen ausmachen sollen.

In unserem Beispiel ist diese Faustregel verletzt, wie bereits aus der mangelnden Anpassung zu erwarten war.

\subsection{Wahl eines passenden Modells}

Für die Wahl eines bestimmten Modells müssen selbstverständlich primär theoretische Uberlegungen herangezogen werden. Sind jedoch noch keine ausgereiften theoretischen Vorstellungen vorhanden, halte ich es für empfehlenswert, zunächst große Variablenblöcke, etwa alle Haupteffekte, dann alle Interaktionseffekte erster Ordnung, dann die zweiter Ordnung usw. auf ihren Erklärungsbeitrag zu überprüfen und dann innerhalb einer Komplexitätsebene ein passendes Modell durch Elimination einzelner Variablen zu suchen. Diese Vorgehensweise wurde bereits in dem Programm LOGIT (Arminger 1976) für loglineare Modelle verwendet. Wir zeigen dies am Beispiel mit Hilfe von Tabelle 4.

Unter der Rubrik Modelle sind jeweils die am Modell beteiligten Variablen angegeben, wobei 
TABELLE 3 Beobachtete und erwartete Werte und standardisierte Fehler im Modell A + B + Z + A.B + B.Z

\section{UNIT OBSERVED OUT OF FITTED RESIDUAL}

\begin{tabular}{|c|c|c|c|c|}
\hline 1 & 16 & 32 & 9.529 & 2.502 \\
\hline 2 & 52 & 96 & 46.76 & 1.069 \\
\hline 3 & 43 & 57 & 41.54 & .4363 \\
\hline 4 & 5 & 16 & 2.942 & 1.328 \\
\hline 5 & 13 & 35 & 16.77 & -1.275 \\
\hline 6 & 17 & 26 & 17.95 & -4.027 \\
\hline 7 & 132 & 383 & 130.2 & 1919 \\
\hline 8 & 640 & 1155 & 652.5 & -.7407 \\
\hline 9 & 607 & 793 & 622.7 & -1.361 \\
\hline 10 & 47 & 217 & 46.63 & $.6160 \mathrm{E}-01$ \\
\hline 11 & 260 & 461 & 256.8 & .3023 \\
\hline 12 & 265 & 364 & 273.8 & -1.070 \\
\hline 13 & 1 & 3 & .8948 & .1328 \\
\hline 14 & 0 & 1 & .7773 & -1.868 \\
\hline 15 & 329 & 845 & 333.1 & -.2877 \\
\hline 16 & 2925 & 4398 & 2876 & 1.553 \\
\hline 17 & 2838 & 3359 & 2826 & .5812 \\
\hline 18 & 242 & 913 & 234.5 & .5660 \\
\hline 19 & 1874 & 2926 & 1892. & -.6969 \\
\hline 20 & 2384 & 2877 & 2344. & 1.917 \\
\hline 21 & 1 & 13 & 4.541 & -2.060 \\
\hline 22 & 9 & 14 & 9.780 & -.4543 \\
\hline 23 & 13 & 15 & 12.52 & .3314 \\
\hline 24 & 100 & 207 & 101.4 & -.1950 \\
\hline 25 & 927 & 1246 & 971.1 & -3.009 \\
\hline 26 & 1022 & 1126 & 1022. & $.7683 \mathrm{E}-03$ \\
\hline 27 & 178 & 617 & 208.4 & -2.590 \\
\hline 28 & 1581 & 2036 & 1575. & .2984 \\
\hline 29 & 2118 & 2420 & 2156. & -2.458 \\
\hline 30 & 10 & 23 & 10.17 & $-.7013 \mathrm{E}-01$ \\
\hline 31 & 39 & 51 & 41.43 & -.8732 \\
\hline 32 & 95 & 109 & 98.50 & -1.135 \\
\hline 33 & 16 & 32 & 18.76 & -.9912 \\
\hline 34 & 143 & 162 & 140.7 & .5377 \\
\hline 35 & 147 & 153 & 145.0 & .7132 \\
\hline 36 & 106 & 199 & 85.47 & 2.940 \\
\hline 37 & 722 & 820 & 709.1 & 1.320 \\
\hline 38 & 908 & 960 & 900.5 & 1.007 \\
\hline 39 & 16 & 23 & 12.40 & 1.507 \\
\hline 40 & 94 & 102 & 90.79 & 1.017 \\
\hline 41 & 209 & 217 & 205.2 & 1.137 \\
\hline
\end{tabular}

A, B, Z die Variablen selbst und A.B, A.Z etc. die Interaktionen sind.

Als Basismodelle fungieren

BM1 - alle Interaktionen sind zugelassen

BM2 - alle Zweier Interaktionen sind zugelassen

BM3 - nur Haupteffekte sind zugelassen

BM4 - nur die Regressionskonstante GM ist zugelassen.

Die einzelnen Variablen bzw. ihre Interaktionen werden jeweils auf das kleinste, sie einschließende Basismodell bezogen.

Die Devianzen geben die Abweichungen der erwarteten von den beobachteten Häufigkeiten an. Die Devianzen sind - wenn die unter dem Modell erwarteten Häufigkeiten nur zufällig von den beobachteten Werten abweichen -- $\chi^{2}$-verteilt mit den jeweils angegebenen Freiheitsgraden.

Einzelne Interaktionen, Variable und Variablengruppen können in ihrer Einflußstärke überprüft werden, indem die Basismodelle ohne die jeweiligen Variablen gerechnet werden. Die Differenz der Devianzen ist - wenn das Basismodell zutrifft und die überprüfte Variable keinen Einfluß hat - wiederum $\chi^{2}$-verteilt mit der neben der Differenz stehenden Zahl von Freiheitsgraden.

Werden die durch die einzelnen Variablen erzeugten Devianzen durch die Gesamtdevianz - die durch BM4 erzeugt wird - dividiert, erhalten wir den Anteil an erklärter Devianz, analog zum Anteil an erklärter Varianz.

Im obigen Beispiel liefert keines der Modelle eine ausreichende Anpassung an die Daten. Dies bedeutet, daß die quantitative Variable $Z$ keinen linearen Zusammenhang mit den logits aufweist:

TABELLE 4 Modelle zur Erklärung der Daten in Tabelle 1

\begin{tabular}{|c|c|c|c|c|c|c|c|}
\hline $\begin{aligned} \text { BM 1: } & A+B+Z+A \cdot B+A \cdot Z+B \cdot Z+A \cdot B \cdot Z \\
\text { BM 2: } & A+B+Z+A \cdot B+A \cdot Z+B \cdot Z \\
& A+B+Z+A \cdot Z+B \cdot Z \\
& A+B+Z+A \cdot B+B \cdot Z \\
& A+B+Z+A \cdot B+A \cdot Z \\
\text { BM }: & A+B+Z \\
& B+Z \\
& A+Z \\
& A+B \\
\text { BM4: } & \text { GM }\end{aligned}$ & $\begin{array}{c}54.08 \\
60.93 \\
109.1 \\
71.73 \\
78.61 \\
163.2 \\
\\
196.8 \\
3.502 .0 \\
883.5 \\
4.469 .0\end{array}$ & $\begin{array}{l}23 \\
27 \\
31 \\
29 \\
29 \\
35\end{array}$ & $\begin{array}{l}\text { A.B.Z } \\
\text { A.B } \\
\text { A.Z } \\
\text { B.Z } \\
\text { A.B,A.Z,B.Z, } \\
\text { A.B.Z } \\
\text { A } \\
\text { B } \\
\text { Z } \\
\quad\end{array}$ & $\begin{array}{l}\text { BM1: } \\
\text { BM2: } \\
\text { BM 2: } \\
\text { BM2: } \\
\text { BM1: } \\
\text { BM3: } \\
\text { BM3: } \\
\text { BM3: }\end{array}$ & $\begin{array}{l}6.85 \\
48.17 \\
10.8 \\
17.68\end{array}$ & $\begin{array}{r}- \\
4 \\
4 \\
2 \\
2 \\
12 \\
2 \\
2 \\
1 \\
-\end{array}$ & $\begin{array}{l}-\overline{0.001} \\
0.011 \\
0.002 \\
0.004\end{array}$ \\
\hline
\end{tabular}


Wir werden das zeigen können, wenn wir das Einkommen als qualitative Variable auffassen.

Die einzelnen Variablen und Interaktionen haben alle signifikanten Einfluß, wenn wir eine Irrtumswahrscheinlichkeit von z.B. $\alpha=0,05$ ansetzen und die Problematik nacheinander durchgeführter statistischer Tests im Moment außer acht lassen. Die Einflußstärke der einzelnen Variablen ist jedoch höchst unterschiedlich, wie die erklärten Devianzen zeigen. Den überragenden Einfluß nimmt die Variable $B$ (Kind) mit $B_{B}=0.748$ ein, wesentlich geringer sind die Stärken von $Z$ (Einkommen des Mannes) mit $\mathrm{B}_{\mathrm{Z}}=0.16$ und $\mathrm{A}$ (Ausbildung) mit $B_{A}=0.024$.

\subsection{Alternative Modelle}

Um die Hypothese, daß der Einfluß von Einkommen nicht linear ist, zu überprüfen, fassen wir das Einkommen als qualitative Variable mit 5 Ausprägungen auf und berechnen das Modell $\mathrm{A}+\mathrm{B}+\mathrm{X}+\mathrm{A}$. $B+B$.X fur die logits, das bis auf $X$ an Stelle von $Z$ dem vorigen Modell entspricht.

DEVIANCE $=31.51>x_{20.0 .95}^{2}=31.4$. Trotzdem weist dieses Modell einen wesentlich höheren An-

TABELLE 5 Parameter und Standardabweichungen im Modell A+B+X+A.B+B.X (Logit Modell)

\begin{tabular}{|c|c|c|c|}
\hline & $\begin{array}{l}\text { DEVIANCE } \\
31.51 \\
\text { ESTIMATE }\end{array}$ & $\begin{array}{l}\text { DF } \\
20 \\
\text { S.E. }\end{array}$ & PARAMETER \\
\hline 1 & .6873 & .1452 & $<\mathrm{GM}$ \\
\hline 2 & -.6216 & $.7775 \mathrm{E}-01$ & $A(2)$ \\
\hline 3 & -.2557 & .2749 & $A(3)$ \\
\hline 4 & 1.361 & .1776 & B (2) \\
\hline 5 & 2.415 & .2008 & B (3) \\
\hline 6 & -.9009 & .1476 & $X(2)$ \\
\hline 7 & -1.122 & .1417 & $X(3)$ \\
\hline 8 & -1.335 & .1627 & $X(4)$ \\
\hline 9 & -.7381 & .3238 & $X(5)$ \\
\hline 10 & .5901 & $.8724 \mathrm{E}-01$ & $A(2)$. B (2) \\
\hline 11 & .4364 & $.9465 \mathrm{E}-01$ & A (2). B (3) \\
\hline 12 & .4095 & .3568 & $\mathrm{~A}(3) . \mathrm{B}(2)$ \\
\hline 13 & .1643 & .3537 & A (3). B (3) \\
\hline 14 & $.4567 \mathrm{E}-01$ & .1811 & B (2). X (2) \\
\hline 15 & -.2717 & .1742 & B (2). X (3) \\
\hline 16 & -.4751 & .1971 & 8 (2). $\times(4)$ \\
\hline 17 & -1.317 & .3811 & B (2). X (5) \\
\hline 18 & $-.3220 \mathrm{E}-01$ & .2031 & $8(3) . \times(2)$ \\
\hline 19 & -.2530 & .1968 & $\mathrm{~B}(3) . \mathrm{X}(3)$ \\
\hline 20 & -.5926 & .2214 & $B(3) . X(4)$ \\
\hline 21 & -1.346 & .4278 & $B(3) . X(5)$ \\
\hline
\end{tabular}

passungsgrad als das vorige auf. Der Unterschied liegt in $X$. Hätte das Einkommen einen linearen Effekt, müßte $X(1)=0>X(2)>X(3)>X(4)$ $>X(5)$ gelten. Tatsächlich ist jedoch $X(5)>$ $X(2)$, d.h. die unterste Einkommensstufe X5 des Mannes hat einen weniger negativen Effekt auf die Nichterwerbstätigkeit der Frau als die zweite Einkommensstufe. Die Interpretation der anderen Variablen bleibt gleich.

Man beachte, daß die Parameter dieses Modells nicht mit ECTA geschätzt werden können, da die oben angegebenen leeren Zellen vorliegen.

TABELLE 6 Beobachtete und erwartete Häufigkeiten und standardisierte Fehler im Modell $A+B+X+A . B+B . X$ (Logit Modell)

UNIT OBSERVED OUT OF FITTED RESIDUAL

\begin{tabular}{|c|c|c|c|c|}
\hline 1 & 16 & 32 & 15.59 & .1439 \\
\hline 2 & 52 & 96 & 47.84 & .8500 \\
\hline 3 & 43 & 57 & 41.88 & .3370 \\
\hline 4 & 5 & 16 & 5.407 & -.2151 \\
\hline 5 & 13 & 35 & 17.16 & -1.408 \\
\hline 6 & 17 & 26 & 18.12 & -.4794 \\
\hline 7 & 132 & 383 & 131.5 & $.4945 \mathrm{E}-01$ \\
\hline 8 & 640 & 1155 & 645.8 & -.3449 \\
\hline 9 & 607 & 793 & 605.9 & $.9338 E-01$ \\
\hline 10 & 47 & 217 & 47.59 & $-9.755 \mathrm{E}-01$ \\
\hline 11 & 260 & 461 & 254.2 & .5450 \\
\hline 12 & 265 & 364 & 265.4 & $-.4355 \mathrm{E}-01$ \\
\hline 13 & 1 & 3 & .8649 & .1722 \\
\hline 14 & 0 & 1 & .7472 & -1.719 \\
\hline 15 & 329 & 845 & 332.1 & -.2176 \\
\hline 16 & 2925 & 4398 & 2894. & .9895 \\
\hline 17 & 2838 & 3359 & 2852. & -.6866 \\
\hline 18 & 242 & 913 & 235.6 & .4864 \\
\hline 19 & 1874 & 2926 & 1904. & -1.181 \\
\hline 20 & 2384 & 2877 & 2370. & .6753 \\
\hline 21 & 1 & 13 & 4.341 & -1.965 \\
\hline 22 & 9 & 14 & 9.684 & -.3961 \\
\hline 23 & 13 & 15 & 12.56 & .3105 \\
\hline 24 & 100 & 207 & 92.49 & 1.051 \\
\hline 25 & 927 & 1246 & 956.0 & -1.943 \\
\hline 26 & 1022 & 1126 & 1011. & 1.123 \\
\hline 27 & 178 & 617 & 186.7 & -.7594 \\
\hline 28 & 1581 & 2036 & 1551. & 1.584 \\
\hline 29 & 2118 & 2420 & 2128 . & -.5955 \\
\hline 30 & 10 & 23 & 8.850 & .4929 \\
\hline 31 & 39 & 51 & 40.47 & -.5096 \\
\hline 32 & 95 & 109 & 96.88 & -.5718 \\
\hline 33 & 16 & 32 & 21.29 & -1.982 \\
\hline 34 & 143 & 162 & 143.5 & -.1213 \\
\hline 35 & 147 & 153 & 146.4 & .2304 \\
\hline 36 & 106 & 199 & 102.8 & .4589 \\
\hline 37 & 722 & 820 & 723.7 & -.1807 \\
\hline 38 & 908 & 960 & 910.8 & -.4035 \\
\hline 39 & 16 & 23 & 13.94 & .8775 \\
\hline 40 & 94 & 102 & 91.84 & .7133 \\
\hline 41 & 209 & 217 & 206.8 & .6999 \\
\hline
\end{tabular}


Wir schätzen nun die gleichen Parameter mit einem linearen Modell an Stelle eines logit-Modells.

Dieses Schätzverfahren ist ebenfalls ein Untermodell des GLM-Ansatzes und ist formal identisch mit dem linearen Ansatz in NONMET.

Die abhängige Variable ist der Anteilswert $\eta_{i}$ $\eta_{\mathrm{i}}=\mathrm{R}_{\mathrm{i}} / \mathrm{N}_{\mathrm{i}} \mathrm{i}=1, \ldots 41$ bzw. $0.5 / \mathrm{N}_{\mathrm{i}}$ für $\mathrm{R}_{\mathrm{i}}=0$ $\eta_{\mathrm{ijk}}=\beta_{\mathrm{o}}+\beta_{\mathrm{i} \delta}^{\mathrm{A} \delta_{\mathrm{i}}^{\mathrm{A}}}+\beta_{\mathrm{j}}^{\mathrm{B} \delta_{\mathrm{j}}^{\mathrm{B}}}+\beta_{\mathrm{k}}^{\mathrm{X}} \delta_{\mathrm{k}}^{\mathrm{X}}+\beta_{\mathrm{i} j \delta_{\mathrm{i}} \delta_{\mathrm{j}}^{\mathrm{A}}}^{\mathrm{B}}+$ $\beta_{\mathbf{j k}}^{\mathrm{BX} \delta \delta_{\mathbf{j}} \delta \mathrm{X}}$

$\eta_{\mathrm{i}}$ ist ein erwarteter Anteil mit Varianz

$\mathrm{V}_{\mathrm{i}}=\left(\eta_{\mathrm{i}}\left(1-\eta_{\mathrm{i}}\right)\right) / \mathrm{N}_{\mathrm{i}}$

Faßt man $\eta_{\mathrm{i}}, \mathrm{i}=1, \ldots .41$, die $\beta_{\mathrm{j}}, \mathrm{j}=1, \ldots .21$ in Vektoren $\eta, \beta$ und die Dummy Variablen in einer Matrix $\bar{X}$ sowie die Varianzen $V_{i}, i=1$, ... 41 in einer Diagonalmatrix $\underline{V}=\operatorname{diag}\left\{V_{i}\right\}$ zusammen, besteht der GSK-Ansatz in der Schätzung von $\beta$ durch eine gewichtete Regression (vgl. Küchler 1978).

$\underline{b}=\hat{\beta}=\left(X^{\prime} \underline{V}^{-1} X\right)^{-1} X^{\prime} V^{-1} \eta$

$\overline{\text { Schreibt } \operatorname{man}} \overline{\mathrm{W}}=\overline{\mathrm{V}}^{-}$erhalten wir

$\mathrm{b}=\left(\mathrm{X}^{\prime} \mathrm{W} X\right)^{-1} \mathrm{X}^{\prime} \underline{\mathrm{W}} \eta$

Als Ergebnis erhalten wir Tabelle 7.

TABELLE 7 Parameter und Standardabweichungen im Modell A+B+X+A.B+B.X (Lineares Modell)

\begin{tabular}{|c|c|c|c|}
\hline & $\begin{array}{l}\text { DEVIANCE } \\
147.8 \\
\text { ESTIMATE }\end{array}$ & $\begin{array}{l}\text { DF } \\
20 \\
\text { S.E. }\end{array}$ & PARAMETER \\
\hline 1 & .6661 & $.6633 \mathrm{E}-01$ & $<\mathrm{GM}$ \\
\hline 2 & -.1370 & $.3659 \mathrm{E}-01$ & $A(2)$ \\
\hline 3 & $-.7237 \mathrm{E}-01$ & .1077 & A (3) \\
\hline 4 & .2158 & $.6772 \mathrm{E}-01$ & B (2) \\
\hline 5 & .3008 & $.6659 \mathrm{E}-01$ & B (3) \\
\hline 6 & -.2229 & $.6788 \mathrm{E}-01$ & $X(2)$ \\
\hline 7 & -.2740 & $.6421 \mathrm{E}-01$ & $X(3)$ \\
\hline 8 & -.3177 & $.7381 E-01$ & $X(4)$ \\
\hline 9 & -.1811 & .1554 & $X(5)$ \\
\hline 10 & .1366 & $.3843 E-01$ & $A(2) . B(2)$ \\
\hline 11 & .1148 & $.3702 \mathrm{E}-01$ & $A(2)$. B (3) \\
\hline 12 & .1091 & .1102 & A (3). B (2) \\
\hline 13 & $.6718 \mathrm{E}-01$ & .1080 & $A(3) . B(3)$ \\
\hline 14 & .1066 & $.6924 \mathrm{E}-01$ & B (2). X (2) \\
\hline 15 & $.4806 \mathrm{E}-01$ & $.6573 \mathrm{E}-01$ & $\mathrm{~B}(2) . \times(3)$ \\
\hline 16 & $-.7084 \mathrm{E}-02$ & $.7798 \mathrm{E}-01$ & B (2). X (4) \\
\hline 17 & -.1966 & .1764 & $B(2) . X(5)$ \\
\hline 18 & .1575 & $.6817 \mathrm{E}-01$ & B (3). X (2) \\
\hline 19 & .1546 & $.6456 \mathrm{E}-01$ & B (3). X (3) \\
\hline 20 & .1093 & $.7589 \mathrm{E}-01$ & $B(3) . \times(4)$ \\
\hline 21 & $-.4782 \mathrm{E}-01$ & .1698 & B (3). X (5) \\
\hline
\end{tabular}

Betrachtet man die standardisierten Residuen, ist die Anpassung erheblich schlechter als im logit-Modell. Dies ist eine Folge der relativ großen Anzahl von relativen Häufigkeiten, die über den Wert 0.8 hinausgehen. Zwischen 0.2 und 0.8 ist - wie in Figur 1 ersichtlich - die Funktion $\pi=\exp (\eta) /(1+\exp (\eta))$ durch eine lineare Funktion gut angenähert.

Interpretiert man die geschätzten Parameter, kommt man zum gleichen Ergebnis wie in der logit-Analyse. Mittlere (A (2) $=-.1370)$ und höhere Ausbildung (A (3) $=-0.07237$ ) verrin-

TABELLE 8 Beobachtete und erwartete Anteilswerte und standardisierte Fehler im Modell A+B+X+A.B+B.X (Lineares Modell)

\begin{tabular}{|c|c|c|c|}
\hline UNIT & OBSERVED & FITTED & RESIDUAL \\
\hline 1 & .5000 & .4850 & .2402 \\
\hline 2 & .5417 & .5042 & 1.087 \\
\hline 3 & .7544 & .7380 & .5808 \\
\hline 4 & .3125 & .3480 & -.3693 \\
\hline 5 & .3714 & .5038 & -.2045 \\
\hline 6 & .6538 & .7158 & -1.128 \\
\hline 7 & .3446 & .3484 & -.1900 \\
\hline 8 & .5541 & .5571 & -.3062 \\
\hline 9 & .7654 & .7585 & .9589 \\
\hline 10 & .2166 & .2114 & .2104 \\
\hline 11 & .5640 & .5567 & .4782 \\
\hline 12 & .7280 & .7363 & -.6781 \\
\hline 13 & .3333 & .2760 & .2580 \\
\hline 14 & $.1000 \mathrm{E}-01$ & .7533 & -7.508 \\
\hline 15 & .3893 & .3920 & -.2058 \\
\hline 16 & .6651 & .6559 & 2.225 \\
\hline 17 & .8449 & .8475 & -1.041 \\
\hline 18 & .2651 & .2550 & .7996 \\
\hline 19 & .6405 & .6555 & -2.828 \\
\hline 20 & .8286 & .8253 & 1.161 \\
\hline 21 & $.7692 \mathrm{E}-01$ & .3197 & -3.419 \\
\hline 22 & .6429 & .6926 & -.6501 \\
\hline 23 & .8667 & .8423 & .7616 \\
\hline 24 & .4831 & .4432 & 1.599 \\
\hline 25 & .7440 & .7656 & -3.450 \\
\hline 26 & .2885 & .3062 & -1.148 \\
\hline 28 & .7765 & .7652 & 2.604 \\
\hline 29 & .8752 & .8792 & -1.699 \\
\hline 30 & .4348 & .3708 & .8235 \\
\hline 31 & .7647 & .8023 & -1.303 \\
\hline 32 & .8716 & .8962 & -2.148 \\
\hline 33 & .5000 & .6661 & -2.657 \\
\hline 34 & .8827 & .8819 & $.9582 \mathrm{E}-0$ \\
\hline 35 & .9608 & .9669 & -1.964 \\
\hline 36 & .5327 & .5291 & .1482 \\
\hline 37 & .8805 & .8815 & -.2538 \\
\hline 38 & .9458 & .9447 & .6680 \\
\hline 39 & .6957 & .5937 & 1.926 \\
\hline 40 & .9216 & .9186 & .4006 \\
\hline 41 & .9631 & .9617 & .5873 \\
\hline
\end{tabular}


gern gegenüber keiner Ausbildung die Wahrscheinlichkeit, nicht erwerbstätig zu sein.

Kinder über $6(\mathrm{~B}(2)=.2158)$ und unter 6 $(B(3)=.3008)$ erhöhen die Wahrscheinlichkeit der Nichterwerbstätigkeit beträchtlich. Das Einkommen zeigt den gleichen nichtlinearen Effekt wie zuvor.

\section{Struktur verallgemeinerter linearer Modelle}

\subsection{Modellformulienung}

Wir zeigen nun lineare, loglineare und logit-Modelle als Spezialfälle verallgemeinerter linearer Modelle auf. Der an den mathematischen Beweisen interessierte Leser sei auf Arminger (1982) verwiesen.

Wir gehen vom einfachen Modell klassischer univariater Regressionsanalyse aus:

$$
\text { (3.1) } \begin{aligned}
\mathrm{y}_{\mathrm{i}} & =\mu_{\mathrm{i}}+\mathrm{e}_{\mathrm{i}}, \mathrm{i}=1, \ldots \mathrm{n} \\
\mathrm{y}_{\mathrm{i}} & \sim \mathrm{N}^{\mathrm{N}}\left(\mu_{\mathrm{i}}, \sigma^{2}\right), \sigma_{\mathrm{ij}}=0 \text { für } \mathrm{i} \neq \mathrm{j} \\
\mu_{\mathrm{i}} & =\sum_{\mathrm{j}=1}^{\mathrm{p}} \beta_{\mathrm{j}} \mathrm{x}_{\mathrm{ij}}, \mathrm{x}_{\mathrm{il}}=1 \\
\mathrm{y}_{\mathrm{i}} & =\text { voneinander stochastisch unabhängige } \\
& \text { normalverteilte Beobachtungen } \\
\mu_{\mathrm{i}} & =\text { Erwartungswert oder systematische } \\
& \text { Komponente } \\
\mathbf{e}_{\mathrm{i}} & =\text { Fehler oder zufällige Komponente } \\
\mathrm{x}_{\mathrm{ij}} & =\text { feste Werte der unabhängigen Variablen } \\
\beta_{\mathrm{j}} & =\text { Regressionskoeffizienten }
\end{aligned}
$$

$\beta_{1}$ wird als Regressionskonstante bezeichnet. Können die $x_{i j}, j=2, \ldots$ p beliebige Werte annehmen, sprechen wir von Regressionsanalyse.

Wenn die $x_{i j}, j=1, \ldots p$ die einzelnen Ausprägungen von qualitativen unabhängigen Variablen in Form von Dummy Variablen darstellen, werden die zugehörigen $\beta_{\mathrm{j}}$ als Haupt- und Interaktionseffekte der Varianzanalyse bezeichnet. Treten sowohl quantitative als auch qualitative unabhängige Variable auf, sprechen wir von Kovarianzanalyse.

In Matrixschreibweise läßt sich (3.1) so ausdrükken:
(3.2) $\frac{\mathrm{y}}{\mathrm{nx} 1}=\frac{\mu}{\mathrm{nxl}}+\frac{\mathrm{e}}{\mathrm{nxl}}, \mathrm{y} \sim \mathrm{N}\left(\frac{\mu, \sigma^{2}}{\eta \mathrm{xl}} \frac{\mathrm{I}}{\mathrm{nxn}}\right)$

$\frac{\mu}{\mathrm{nxl}}=\frac{\mathrm{X}}{\mathrm{nxp}} \frac{\beta}{\mathrm{pxl}}$

Die Matrix X wird bei Vorliegen von Dummy Variablen auch als Designmatrix bezeichnet. $X$ muß von vollem $R$ ang, also $R g \underline{X}=p$, sein, da sonst keine eindeutige Lösung für $\beta$ existiert. Bekanntlich fallen bei (3.2) Maximum Likelihood-Schätzung und Kleinste-Quadrate-Lösung zusammen, so daß gilt:

(3.3) $\underline{\hat{\beta}}=\underline{b}=\left(X^{\prime} X\right)^{-1} \underline{X}^{\prime} \underline{y}$

(3.4) $\underline{\underline{b}} \sim \bar{N}\left(\underline{\beta}, \bar{\sigma}^{2}(\underline{X} \underline{X})^{-1}\right)$ exakt

Aus (3.4) lassen sich die üblichen t- und F-Tests der Regressions- bzw. (Ko)varianzanalyse ableiten.

In der Theorie verallgemeinerter linearer Modelle wird (3.1) in zweierlei Hinsicht erweitert. An die Stelle der Normalverteilung von $y_{i}$ tritt die einparametrige exponentielle Familie.

$$
\begin{aligned}
& y_{i}, i=1, \ldots n \text { stochastisch unabhängig } \\
& y_{i}=\mu_{i}+e_{i} \text { mit E } y_{i}=\mu_{i}, V\left(y_{i}\right)=\sigma_{i}^{2} \\
& f\left(y_{i}\right)=\exp \left\{\left[y_{i} \theta_{i}-b\left(\theta_{i}\right),\right] / a_{i}(\phi)+\right. \\
& \left.c\left(y_{i}, \phi\right)\right\} \\
& \mathrm{V}\left(\mathrm{y}_{\mathrm{i}}\right)=\text { Varianz von } \mathrm{y}_{\mathrm{i}} \\
& \mathrm{f}\left(\mathrm{y}_{\mathrm{i}}\right)=\text { Dichtefunktion von } \mathrm{y}_{\mathrm{i}} \\
& \mathrm{b}\left(\theta_{\mathrm{i}}\right), \mathrm{a}_{\mathrm{i}}(\phi), \mathrm{c}\left(\mathrm{y}_{\mathrm{i}}, \phi\right) \text { sind Funktionen, } \\
& \text { die gewisse Regula- } \\
& \text { ritätsbedingungen } \\
& \text { erfülen }
\end{aligned}
$$

Zwischen den Erwartungswert $\mu_{i}$ und den linearen Prädiktor

$$
\sum_{j=1}^{p} \beta_{j} x_{i j}
$$

tritt eine Verbindungs- (link) Funktion g.

$$
\begin{aligned}
\eta_{\mathrm{i}} & =\mathrm{g}\left(\mu_{\mathrm{i}}\right), \mu_{\mathrm{i}}=\mathrm{g}^{-1}\left(\eta_{\mathrm{i}}\right)=\mathrm{h}\left(\eta_{\mathrm{i}}\right) \\
\eta_{\mathrm{i}} & =\sum_{\mathrm{j}=1}^{\mathrm{p}} \beta_{\mathrm{j}} \mathrm{x}_{\mathrm{ij}}
\end{aligned}
$$

$\mathrm{g}$ ist monoton und zweimal differenzierbar. 
(3.5) und (3.6) lassen sich kompakt darstellen mit

$$
\text { (3.7) } \begin{aligned}
\frac{\mathrm{y}}{\mathrm{nx}} & =\frac{\mu}{\mathrm{nx}}+\frac{\mathrm{e}}{\mathrm{nx}}, \eta_{\mathrm{i}}=\mathrm{g}\left(\eta_{\mathrm{i}}\right), \\
\frac{\eta}{\mathrm{n} \times \mathrm{l}} & =\frac{\mathrm{x}}{\mathrm{nxl}} \frac{\beta}{\mathrm{pxI}}
\end{aligned}
$$

\subsection{Eigenschaften und spezielle Verteilungen der exponentiellen Familie}

Es läßt sich zeigen, daß allgemein für die exponentielle Familie gilt:

$E\left(y_{i}\right)=\mu_{i}=b^{\prime}\left(\theta_{i}\right)=\frac{d b\left(\theta_{i}\right)}{d \theta_{i}}$

$V\left(y_{i}\right)=b^{\prime \prime}\left(\theta_{i}\right) a_{i}(\phi)$

b" $\left(\theta_{i}\right)$ wird als Varianzfunktion bezeichnet.

(3.8) und (3.9) stellen die Verbindung zwischen $\mu_{i}, V\left(y_{i}\right)$ und $\theta_{i}, \phi$ her.

In Tabelle 9 sind einige wichtige Spezialfälle der einparametrigen exponentiellen Familie angegeben.

\subsection{Link-Funktionen}

Für die obigen Verteilungen bieten sich als linkFunktionen solche Funktionen an, in denen der lineare Prädiktor gleich dem kanonischen Parameter $\theta$ gesetzt wird.

TABELLE 9 Einige spezielle Dichtefunktionen der exponentiellen Familie ( $m$ ist die Stichprobengröße und

\begin{tabular}{|c|c|c|c|c|}
\hline & Normal & Poisson & Binomial & Gamma \\
\hline $\begin{array}{l}\text { Wertebereich } \\
\text { von y }\end{array}$ & $(-\infty, \infty)$ & $0,1,2$. & $(0,1)$ & $(0, \infty)$ \\
\hline$a(\phi)$ & $\phi$ & 1 & $\frac{1}{m}$ & $\phi$ \\
\hline$b(\theta)$ & $\frac{1}{2} \theta^{2}$ & $e^{\theta}$ & $\ln \left(1+e^{\theta}\right)$ & $\ln \theta$ \\
\hline$c(y, \phi)$ & $\frac{1}{2}\left(y^{2}+\right.$ & $-\ln y$ & $\ln \left(\left(_{m y}^{m}\right)+\right.$ & $\begin{array}{l}\left(\phi^{-1}-1\right) \ln y \\
+\phi^{-1} \ln \phi^{-1} \\
-\ln \Gamma\left(\phi^{-1}\right)\end{array}$ \\
\hline$\mu=E y$ & $\theta$ & $e^{\theta}$ & $e^{\theta} /\left(1+e^{\theta}\right)$ & $\frac{1}{9}$ \\
\hline $\begin{array}{l}\text { Vurianzfunktion } \\
b^{\prime \prime}(\theta)\end{array}$ & 1 & $\mu$ & $\mu(1-\mu)$ & $-\mu^{2}$ \\
\hline
\end{tabular}
$y$ ist der beobachtete Anteil in der Binomialverteilung)
Normalverteilung: $\eta=\theta=\mu$ also $g(\mu)=$ id $\mu$ Poissonverteilung: $\eta=\theta=\ln \mu$ also $\mathrm{g}(\mu)=\ln \mu$ Binomialvertei-

lung:

$$
\eta=\theta=\ln (\mu /(1-\mu) \text { also } \mathrm{g}
$$$$
(\mu)=\ln (\mu /(1-\mu))
$$

$\Gamma$.Verteilung: $\quad \mu=\theta=1 / \mu$ also $\mathrm{g}(\mu)=1 / \mu$

Da sich ein multinomial verteilter Zufallsvektor, der z.B. eine Kontingenztabelle beschreibt, als Produkt unabhängiger poissonverteilter Zufallsvariablen darstellen läßt (Birch 1963), ist mit der link-Funktion $\eta=\ln \mu$ und der Poissonverteilung die Klasse der loglinearen Modelle definiert.

Mit der link-Funktion $\eta=\ln (\mu /(1-\mu))$ und der Binomialverteilung ist die Klasse der logit Modelle beschrieben.

Es ist nicht erforderlich, $\eta=\theta$ zu setzen. Gegenbeispiele sind etwa bei der Binomialverteilung die links

probit: $\eta=\phi^{-1}(\mu)$. $(\phi=$ Verteilungsfunktion der $N(0,1)$ Verteilung)

Komplementäre $\log \log$ Funktion: $\eta=\ln (-\ln$ $(1-\mu))$.

\subsection{Designmatrix $X$, fehlende Beobachtungen, Freiheitsgrade}

Wie im einfachen Modell (3.1) können auch im GLM Ansatz Dummy Variable auftreten. Ebenso muB $X$ von vollem Spaltenrang sein. Zum allgemeinen Aufbau von Designmatrizen sei auf Bock (1975) und Küchler (1979) verwiesen.

Wir merken an dieser Stelle nur folgende Punkte an:

In GLIM wird die Reparametrisierung der cornered effects verwendet; der Haupteffekt der ersten Ausprägung jeder qualitativen Variabeln wird 0 gesetzt. Die Haupteffekte aller anderen Ausprägungen sind daher als Differenzen zur ersten Ausprägung zu interpretieren. Formal wird dies dadurch erzeugt, daB - wie im Beispiel angegeben - die Dummy Variable $\delta_{1}$ der ersten Ausprägung immer 0 ist. Da die Dummy Variablen der Interaktionseffekte als Produkte der Dummy Variablen der Haupteffekte gebildet werden, sind auch die entsprechenden Interaktionseffekte, an denen eine erste Ausprägung beteiligt ist, gleich 0 . Die „cornered effects“ lassen sich leicht auf die gewohnten zentrierten Effekte umrechnen und umgekehrt. Wie in 
NONMET können auch in GLIM konditionale Effekte mit speziellen Dummy Variablen beschrieben werden.

Sowohl bei der klassischen Varianzanalyse als auch in Kontingenztabellen treten häufig Kombinationen auf, für die Beobachtungen fehlen. Wir bezeichnen Kombinationen, die aus inhaltlichen oder logischen Gründen nicht auftreten können, als fehlende Zellen oder strukturelle Nullen. Ist die Kombination inhaltlich sinnvoll, aber die Stichprobe zu klein, wird die fehlende Beobachtung als Stichproben-Null bezeichnet. In beiden Fällen können die fehlenden Beobachtungen dazu führen, daß trotz Reparametrisierung der Rang $\frac{X}{n \times p}=k<p$ ist.

Um wieder $\mathrm{zu}$ einer eindeutig bestimmten Schätzung zu gelangen, müssen zusätzliche, nämlich $\mathbf{p}-\mathbf{k}$ lineare Restriktionen eingefuhrt werden.

Inhaltlich bedeutet das, daß nicht alle gewünschten $\mathrm{p}$ Parameter $\beta_{1}-\beta_{\mathrm{p}}$ geschätzt werden können, da zu wenige Informationen vorliegen.

Beispiel: Wir gehen von der Kontingenztabelle

\begin{tabular}{c|c|c|c|}
\multicolumn{1}{c}{} & \multicolumn{1}{c}{$B_{1}$} & \multicolumn{1}{c}{$B_{2}$} & \multicolumn{1}{c}{$B_{3}$} \\
\cline { 2 - 4 }$A_{1}$ & $y_{11}$ & $y_{12}$ & $y_{13}$ \\
\cline { 2 - 4 }$A_{2}$ & $y_{21}$ & $y_{22}$ & $y_{23}$ \\
\cline { 2 - 4 } & & &
\end{tabular}

aus, die bei Reparametrisierung durch cornered effects im saturierten Modell wie folgt beschrieben wird:

$$
\begin{aligned}
& \mathrm{Ey}_{\mathrm{jk}}=\mu_{\mathrm{jk}}, \eta_{\mathrm{jk}}=\ln \mu_{\mathrm{jk}}=\beta_{\mathrm{o}}+\beta_{\mathrm{j}}^{\mathrm{A}}+\beta_{\mathrm{k}}^{\mathrm{B}}+\beta_{j \mathrm{j}}^{\mathrm{AB}} \\
& \text { mit } \beta_{1}^{\mathrm{A}}=\beta_{1}^{\mathrm{B}}=\beta_{11}^{\mathrm{AB}}=\beta_{12}^{\mathrm{AB}}=\beta_{13}^{\mathrm{AB}}=\beta_{21}^{\mathrm{AB}}=0
\end{aligned}
$$$$
\left[\begin{array}{l}
\eta_{11} \\
\eta_{12} \\
\eta_{13} \\
\eta_{21} \\
\eta_{22} \\
\eta_{23}
\end{array}\right]=\left[\begin{array}{llllll}
\beta_{0} & \beta_{2}^{\mathrm{A}} & \beta_{2}^{\mathrm{B}} & \beta_{3}^{\mathrm{B}} & \beta_{22}^{\mathrm{AB}} & \beta_{23}^{\mathrm{AB}} \\
1 & 0 & 0 & 0 & 0 & 0 \\
1 & 0 & 1 & 0 & 0 & 0 \\
1 & 0 & 0 & 1 & 0 & 0 \\
1 & 1 & 0 & 0 & 0 & 0 \\
1 & 1 & 1 & 0 & 1 & 0 \\
1 & 1 & 0 & 1 & 0 & 1
\end{array}\right]\left[\begin{array}{c}
\beta_{0} \\
\beta_{2}^{\mathrm{A}} \\
\beta_{2}^{\mathrm{B}} \\
\beta_{3}^{\mathrm{B}} \\
\beta_{22}^{\mathrm{AB}} \\
\beta_{23}^{\mathrm{AB}}
\end{array}\right]
$$

Wenn nun eine Beobachtung fehlt, z.B. $y_{12}$, wird die entsprechende Zeile von $\eta$ und $X$ gestrichen; die Matrix $X$ ohne die zweite Zeile ist dann

$\frac{\mathrm{X}^{*}}{5 \times 6}=\left[\begin{array}{llllll}1 & 0 & 0 & 0 & 0 & 0 \\ 1 & 0 & 0 & 1 & 0 & 0 \\ 1 & 1 & 0 & 0 & 0 & 0 \\ 1 & 1 & 1 & 0 & 1 & 0 \\ 1 & 1 & 0 & 1 & 0 & 1\end{array}\right]$

Die 5. Spalte - die mit $\beta_{22}^{\mathrm{AB}}$ multipliziert wird - ist dann identisch mit der 2 . Spalte - die mit $\beta_{2}^{\mathrm{B}}$ multipliziert wird. Der Rang der Matrix ist daher Rang $\underline{X}^{*}=5$. Als Folge der fehlenden Beobachtung können wir $\beta_{22}^{\mathrm{AB}}$ nicht mehr schätzen, wir setzen diesen Wert daher $=0$. Im Programm. system GLIM wird ein Wert, der auf Grund fehlender Beobachtungen nicht mehr berechnet werden kann, als ALIASED ausgewiesen.

Liegt eine Matrix $\underline{X}$ vor oder wird sie mit Hilfe der oben genannten Produkte aus den Spalten für die Haupteffekte aufgebaut, muß für jede Spalte - etwa durch Berechnung von $\left|\underline{X^{\prime}} \underline{X}\right|$ geprüft werden, ob sie von den vorhergehenden Spalten auf Grund fehlender Beobachtungen linear abhängig ist, oder nicht. Liegt lineare $A b$ hängigkeit vor, wird diese Spalte weggelassen und der entsprechende Parameter $=0$ gesetzt. Diese Prozedur wird sowohl in GLIM als auch in ALMO von Holm (1979) durchgefürt, fehlt aber sowohl in ECTA als auch in NONMET.

Bei der Berechnung der Freiheitsgrade müssen bei fehlenden Beobachtungen die Koeffizienten, die nicht geschätzt werden können, von der Zahl aller Koeffizienten abgezogen werden. Die Anzahl DF der Freiheitsgrade ist daher gegeben durch

$$
\begin{array}{ll}
\mathrm{DF} & =\mathrm{n}-\mathrm{p} \\
\mathrm{n} & =\text { Anzahl der Beobachtungen } \\
\mathrm{p} & =\text { Rang von } \underline{\mathrm{X}}
\end{array}
$$

Diese Formulierung erspart bei loglinearen Modellen die komplizierte Berechnung der Freiheitsgrade bei fehlenden Zellen durch die Berücksichtigung von Marginaltabellen, wie sie etwa noch bei Langeheine (1979) empfohlen wird.

\section{Schätzen und Testen im GLM-Ansatz}

\subsection{ML-Schätzung von $\underline{\beta}$}

Wir maximieren die log likelihood-Funktion 
(4.1) $\mathrm{L} \underline{(\boldsymbol{\beta})}=\sum_{\mathrm{i}=1}^{\mathrm{n}}\left(\mathrm{y}_{\mathrm{i}} \theta_{\mathrm{i}}-\mathrm{b}\left(\theta_{\mathrm{i}}\right)\right) / \mathrm{a}_{\mathrm{i}}(\phi)+\mathrm{c}\left(\mathrm{y}_{\mathrm{i}}, \phi\right)$

in Abhängigkeit von $\beta_{\mathrm{j}}, \mathrm{j}=1, \ldots \mathrm{p}_{\mathrm{j}}$ indem wir den Vektor der ersten Ableitungen $\frac{\partial \mathrm{L}}{\partial \beta}$ sowie die Matrix der zweiten Ableitungen

$\frac{\partial^{2} L}{\partial \underline{\beta} \partial \underline{\beta}}$, bilden und $\frac{\partial L}{\partial \underline{\beta}}=\underline{0}$ setzen.

Das als Optimierungsverfahren verwendete Fisher's scoring ergibt folgenden Algorithmus zur Berechnung von $\underline{b}=\underline{\beta}$, der als iterative gewichtete Regression aufgefaßt werden kann. $q=0,1 \ldots$ ist der Laufindex der Iteration

$$
\begin{aligned}
& \underline{b}^{q+1}=\left(\underline{X}^{\prime} \underline{W} \underline{X}\right)^{-1} \underline{X}^{\prime} \underline{W}^{q}\left(\underline{\eta}^{q}+\underline{r}^{q}\right) \\
& \underline{\eta}^{q}==\underline{X b}^{q} \\
& \underline{r}^{q}=\left(r_{i}^{q} i=1, \ldots \text { mit } r_{i}^{q}=\right. \\
& \left(y_{i}-\mu_{i}^{q}\right) \frac{d n_{j} q}{d \mu_{i} q} \\
& \mu_{i}^{q}=g^{-1}\left(\eta_{i}^{q}\right) \\
& \underline{W q}=\operatorname{diag}\left\{w_{i}^{q}\right\}_{i}=1, \ldots n \\
& w_{i}^{q}=\frac{1}{V^{4}\left(y_{i}\right)}\left(\frac{d \mu_{i}^{q}}{d \eta_{i}^{q}}\right)^{2}
\end{aligned}
$$

Die Iteration wird beendet, sobald

$\sum_{j=1}^{p}\left|b_{j} q+1-b_{j} q\right|<\epsilon, z . B . \epsilon=0.0001$

Spezialfälle:

I) Normalverteilung für Anteilswerte $p_{i}$ bei Stichprobengröße $m_{i}$ in Gruppe $i$.

$p_{i}=\pi_{i}+e_{i}, p_{i} \sim N\left(\pi_{i}, V\left(p_{i}\right)\right), V\left(p_{i}\right)=\left(\pi_{i}\left(1-\pi_{i}\right)\right) / m_{i}$

$\eta_{i}^{q}=\pi_{i}^{q}, \eta_{i}^{q}=\sum_{j=1}^{p} b_{j}^{q} x_{i j}$

$\frac{\mathrm{d} \eta_{i}^{q}}{\mathrm{~d} \pi_{i}^{q}}=1 \Rightarrow \eta_{i}^{q}+r_{i}^{q}=\pi_{i}^{q}+p_{i}-\pi_{i}^{q}=p_{i}$

$W_{i}^{q}=\frac{1}{V^{q}\left(p_{i}\right)}=m_{i} /\left(\pi_{i}^{q}\left(1-\pi_{i}^{q}\right)\right)$

Diese Zuordnung definiert für $\mathrm{q}=0$ genau den GSK-Ansatz für das lineare Modell.

II) Binomialverteilung, logit-Funktion für Anteilswerte $p_{i}$ bei Stichprobengröße $m_{i}$ in Gruppe i.

$p_{i}=\pi_{i}+e_{i}, V\left(p_{i}\right)=\pi_{i}\left(1-\pi_{i}\right) / m_{i}$

$\eta_{i}^{q}=\ln \left(\pi_{i}^{q} / 1-\pi_{i}^{q}\right)$, $\frac{\mathrm{d} \eta_{i}^{q}}{\mathrm{~d} \pi_{i}^{q}}=\frac{1}{\pi_{i}^{q}\left(1-\pi_{i}^{q}\right)}, \frac{\mathrm{d} \pi_{i}^{q}}{\mathrm{~d} \eta_{i}^{q}}=\pi_{i}^{q}\left(1-\pi_{i}^{q}\right)$

$w_{i}^{q}=m_{i} / \pi_{i}^{q}\left(1-\pi_{i}^{q}\right)$

Diese Zuordnung definiert für $q=0$ das logitModell im GSK-Ansatz, in dem allerdings im Gegensatz zum GLM-Ansatz die Iteration nach dem ersten Schritt abgebrochen wird. Die gewichtete Regression in NONMET ist daher identisch mit dem ersten Iterationsschritt im GLMAnsatz.

Im Unterschied zu dem in ECTA verwendeten Iterationsverfahren werden in diesem Algorithmus sofort die Koeffizienten $\beta_{j}$ geschätzt $\left(\lambda_{j}\right.$ in Goodman Terminologie), in ECTA werden nacheinander die das Modell definierenden Randverteilungen iterativ angepaßt und dann erst die Parameter $\lambda_{j}$ berechnet.

\subsection{Tests und Konfidenzintervalle für $\underline{\beta}$}

Auf Grund allgemeiner Ergebnisse der ML-Schätzung gilt unter Regularitätsbedingungen

(4.3) $\underline{b} \sim \underline{N}\left(\underline{\beta},\left(\underline{X}^{\prime} \underline{W X}\right)^{-1}\right)$ asymptotisch

Ist $\underline{c}=\left(c_{j}\right) j=1, \ldots p$ ein linearer Kontrast, also

$\sum_{j=1}^{p} c_{j}=0$, dann gilt asymptotisch

$$
\begin{aligned}
\text { (4.4) } \underline{c}^{\prime} \underline{b} \sim N\left(\underline{c}^{\prime} \underline{\beta}, \underline{c}^{\prime}(\underline{X} \underline{W} \underline{X})^{-1} \underline{c}\right) \\
\left(c^{\prime} b\right)^{2} / \underline{c^{\prime}} \underline{\underline{s}} \sim x_{1}^{2} \text { wenn } H_{0}: \underline{c}^{\prime} \underline{\beta}=0 \mathrm{zu}- \\
\text { trifft. } \underline{\hat{\Sigma}}=\left(X^{\prime} W X\right)^{-1}
\end{aligned}
$$

Dies ist die Grundlage für die im Beispiel verwendeten Konfidenzintervalle und Tests.

\subsection{Güte der Anpassung, Analoga zu partiellen Bestimmtheitsmaßen und Residuen}

Unser Ziel ist es, die $\mathbf{n}$ Beobachtungen $y_{i}$ durch wenige Variable $x_{i j}, j=1, \ldots p$ zu erklären.

$\mu_{\mathrm{i}}=\mu$, also $\mathrm{p}=1$, ist das Minimalmodell $\mathbf{M}_{\mathrm{m}}$ $\mu_{i}=y_{i}$, also $p=n$, ist das saturierte Modell $M_{f}$ 
$1<\mathrm{p}<\mathrm{n}$ definiert das laufende Modell $\mathrm{M}_{\mathrm{c}}$

Zur Uberprüfung, ob ein Modell den Daten hinreichend angepaßt ist, wird generell der Likelihood Ratio-Test verwendet:

Trifft $H_{0}: \beta_{p+1}=\beta_{p+2}=\ldots \beta_{n}=0 z u-$ unterscheiden sich also $M_{c}$ und $M_{f}$ nur zufälig

- ist die LR-Teststatistik

$S(c, f)=-2\left(L\left(M_{c}\right)-L\left(M_{f}\right)\right)$

(4.5) $S(c, f)=2 \sum_{i=1}^{n} \frac{1}{a_{i}(\phi)}\left[y_{i}\left({ }_{r} \theta_{i}-{ }_{c} \theta_{i}\right)+b\left({ }_{c} \theta_{i}\right)\right.$

$\left.-b\left(f \theta_{i}\right)\right]$

asymptotisch $\chi^{2}$-verteilt mit $n-p$ df.

Spezialfall Normalverteilung:

$S(c, f)=\frac{1}{\sigma^{2}} \quad \sum_{i=1}^{n}\left(y_{i}-c \mu_{i}\right)^{2}=\frac{1}{\sigma^{2}} \quad$ SSE

ist unter $H_{o}$ exakt $\chi^{2}$-verteilt mit $n-p$ df.

$S(c, f)$ heißt skalierte Devianz.

Für Modelle mit $a_{i}(\phi)=\phi / W_{i}, W_{i}$ bekannte Gewichte, enthält $D(c, f)=S(c, f)($ Devianz) keine Unbekannten mehr.

Spezialfälle:

Normalverteilung: $\quad D(c, f)=\sum_{i=1}^{n}\left(y_{i}-{ }_{c} \mu_{i}\right)^{2}=S S E$

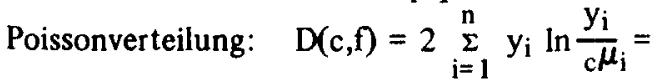

$$
\mathrm{G}^{2}=2 \mathrm{I}=\mathrm{S}(\mathrm{c}, \mathrm{f})
$$

Binomialverteilung: $D(c, f)=2 \underset{j=1}{n} m_{i} y_{i} \ln \frac{y_{j}}{c \mu_{i}}+$

$$
\left(m_{i}-m_{i} y_{i}\right) \ln \frac{1-y_{i}}{1-c \mu_{i}}=S(c, f)
$$

Hat man ein passendes Modell $M_{c 2}$ mit Parameter $\left\{\beta_{1} \ldots \beta_{\mathrm{p}}\right\}$ gefunden, lassen sich sofort Tests zur Úberprïifung, ob einzelne oder mehrere Parameter $\beta_{\mathrm{j}} \epsilon\left\{\beta_{\mathrm{I}} \ldots \beta_{\mathrm{p}}\{\right.$ gleich 0 sind, mit folgendem Likelihood Ratio-Test durchführen.

Sei $\mathbf{M}_{\mathbf{c}_{1}} \subset \mathrm{M}_{\mathbf{c}_{2}}$, d.h. $\left\{\beta_{1} \ldots \beta_{\mathrm{k}}\right\} \mathrm{M}_{\mathrm{c}_{1}} \subset$ $\left\{\beta_{1} \ldots \beta_{\mathbf{p}}\right\} \mathbf{M}_{\mathrm{c}_{2}}$, so ist

(4.6) $S\left(c_{1}, c_{2}\right)=S\left(c_{1}, f\right)-S\left(c_{2}, f\right)$ asymptotisch $\chi_{p-k}^{2}$-verteilt unter $\mathrm{H}_{0}: \beta_{\mathrm{k}+1} \ldots$

$\beta_{\mathrm{n}}=0$

Spezialfall Normalverteilung:

$S\left(c_{1}, c_{2}\right)=\frac{1}{\sigma^{2}} \sum_{j=1}^{n}\left({ }_{c} \mu_{i}-{ }_{c} \mu_{2}\right)=\frac{1}{\sigma^{2}} S S R$ für $M_{c_{1}}$

Minimalmodell

(4.6) ermöglicht das Aufstellen einer Tabelle zur Zerlegung der Devianz analog zur Varianzanalyse (ANODEV statt ANOVA?), wie sie in Tabelle 4 angegeben wurde.

Analog zu quadrierten multiplen partiellen Korrelationskoeffizienten läßt sich ein PRD-Koeffizient definieren $(P R D=$ Proportional Reduction of Deviance), der als Spezialfalle den PRE-Koeffizienten und die $\eta^{2}$-Koeffizienten von Arminger (1979) beinhaltet und gleich wie ein PRE-Koeffizient interpretiert wird.

$$
\begin{aligned}
& P R D=\left(D\left(c_{1}, f\right)-D\left(c_{2}, f\right)\right) / D\left(c_{1}, f\right)= \\
& D\left(c_{1}, c_{2}\right) / D\left(c_{1}, f\right)
\end{aligned}
$$

Für die Analyse von Residuen $\mathrm{e}_{\mathrm{i}}=\mathrm{y}_{\mathrm{i}}-\mu_{\mathrm{i}}$ verwenden wir die standardisierten Pearson-Residuen:

$$
p^{e_{i}}=\left(y_{i}-\mu_{i}\right) / \sqrt{V\left(y_{i}\right)}
$$

$p e_{i} \sim N(0,1)$ asymptotisch bei korrekt spezifiziertem Modell

Spezialfall Poissonverteilung:

(4.9) $\mathrm{p}_{\mathrm{i}}=\left(\mathrm{y}_{\mathrm{i}}-\hat{\mu}_{\mathrm{i}}\right)^{2} / \hat{\mu}_{\mathrm{i}}$ $x^{2}=\sum_{1=1}^{n} p e_{i}^{2}$ ist die Testgröße des $\chi^{2}$.

Anpassungstests.

\section{Polytome abhängige Variable}

Ein spezielles Problem stellen häufig Modelle für abhängige nominale Variable mit mehr als zwei Kategorien dar.

Es gibt hier mehrere Möglichkeiten, dieses Analogon zur multivariaten Regression zu betrachten. Sie werden in der Regel unter der Rubrik „multivariate logits" abgehandelt, da sie Verall- 
gemeinerungen der in 2.3 eingeführten logits darstellen.

Eine besonders einfache Möglichkeit, multivariate logits $\mathrm{zu}$ definieren, wollen wir an folgendem Beispiel demonstrieren:

Wir nehmen an, daß eine abhängige qualitative Variable $\mathrm{C}$ mit Ausprägungen $\mathrm{C}_{\mathrm{k}}, \mathrm{k}=1, \ldots \mathrm{K}$ von zwei unabhängigen qualitativen Variablen $A$ mit $A_{i}, i=1 \ldots l$ und $B$ mit $B_{j}, j=1 \ldots 1$ beeinflußt wird. Die Häufigkeiten der Kombinationen $A_{i} B_{j} C_{k}$ sind in einer Kontingenztabelle $\{A B C\}$ zusammengefaßt. Wir nehmen an, daB die zweidimensionalen Randverteilungen $\{\mathrm{AB}\}$, $\{A C\},\{B C\}$ eine gute Anpassung an $\{A B C\}$ erzeugen. Dem entspricht das loglineare Modell

$y_{i j k}=$ Häufigkeit von $A_{i} B_{j} C_{k}$

E $y_{i j k}=\mu_{i j k}$

$\eta_{\mathrm{ijk}}=\ln \mu_{\mathrm{ijk}}=\beta_{\mathrm{o}}+\beta_{\mathrm{i}}^{\mathrm{A}}+\beta_{\mathrm{j}}^{\mathrm{B}}+\beta_{\mathrm{k}}^{\mathrm{C}}+\beta_{\mathrm{ij}}^{\mathrm{AB}}+$ $\beta_{\mathrm{ik}}^{\mathrm{AC}}+\beta_{\mathrm{jk}}^{\mathrm{BC}}$

In Parametern lautet das Modell

$\eta_{i j k}=\ln \mu_{\mathrm{ijk}}=\beta_{\mathrm{o}}+\beta_{\mathrm{i}}^{\mathrm{A}}+\beta_{\mathrm{j}}^{\mathrm{B}}+\beta_{\mathrm{k}}^{\mathrm{C}}+\beta_{\mathrm{ij}}^{\mathrm{AB}}+$

$\beta_{i k}^{A C^{\prime}}+\beta_{j k}^{B C}$

Wenn wir $C$ als abhängige Variable betrachten, können wir $z . B$. jede Kategorie $C_{k} k=2, \ldots K$ mit der ersten Kategorie $C_{1}$ vergleichen.

(5.1) $\ln \left(\mu_{\mathrm{ijk}} / \mu_{\mathrm{ijl}}\right)=\eta_{\mathrm{ijk}}-\eta_{\mathrm{ijl}}=\beta_{\mathrm{o}}+\beta_{\mathrm{i}}^{\mathrm{A}}+$ $\beta_{j}^{B}+\beta_{k}^{C}+\beta_{i j}^{A B}+\beta_{i k}^{A C}+\beta_{j k}^{B C}-\beta_{o}-\beta_{i}^{A}-\beta_{j}^{B}-$

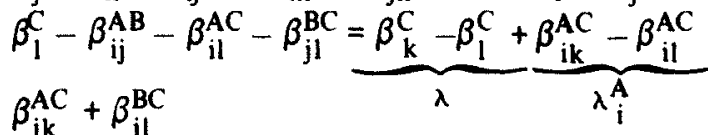
$\frac{\beta_{j k}^{A C}+\beta_{j l}^{B C}}{\lambda_{j}^{B}}$

$=\lambda+\lambda_{i}^{A}+\lambda_{j}^{B}$

Diese Darstellung ist besonders günstig, wenn wir für das loglineare Modell cornered effects als Reparametrisierung gewählt haben. $\mathrm{Da}$ in diesem Fall $\beta_{1}^{C}=\beta_{\mathrm{il}}^{\mathrm{AC}}=\beta_{\mathrm{jl}}^{\mathrm{BC}}=0$ sind, gilt

(5.2) $\eta_{\mathrm{ijk}}-\eta_{\mathrm{ijl}}=\beta_{\mathrm{k}}^{\mathrm{C}}+\beta_{\mathrm{ik}}^{\mathrm{AC}}+\beta_{\mathrm{jk}}^{\mathrm{BC}} \mathrm{k}=2, \ldots \mathrm{K}$
Wir erläutern die Interpretation wiederum an dem in 2. angefuhrten Beispiel. Die Variablen A (Ausbildung der Frau), B (Kinder) und C (Art der Beschäftigung) sind wie oben definiert. Im Unterschied zu vorher betrachten wir jetzt

C mit allen drei Ausprägungen als abhängige Variable

C1 Nicht erwerbstätig

C2 Un/angelernte Arbeiterin

C3 Ausfuihrende Angestellte und Beamte

Um die Tabelle zu vereinfachen, lassen wir das Einkommen des Mannes als unabhängige Variable unberücksichtigt.

Die Tabelle der einzelnen Kombinationen für A,B,C hat folgende Gestalt (Tabelle 10):

TABELLE 10 Art der Erwerbstätigkeit von Frauen $\mathbf{N}=$ Häufigkeit in der Kom bination $A B C$

\begin{tabular}{rrrr}
\hline A & B & C & \multicolumn{1}{r}{ N } \\
\hline 1 & 1 & 1 & 593 \\
1 & 1 & 2 & 731 \\
1 & 1 & 3 & 175 \\
1 & 2 & 1 & 4697 \\
1 & 2 & 2 & 2045 \\
1 & 2 & 3 & 325 \\
1 & 3 & 1 & 4657 \\
1 & 3 & 1 & 713 \\
1 & 3 & 3 & 118 \\
2 & 1 & 1 & 478 \\
2 & 1 & 2 & 190 \\
2 & 1 & 3 & 1194 \\
2 & 2 & 1 & 4450 \\
2 & 2 & 2 & 609 \\
2 & 2 & 3 & 1324 \\
2 & 3 & 1 & 5692 \\
2 & 3 & 2 & 247 \\
2 & 3 & 3 & 708 \\
3 & 1 & 1 & 28 \\
3 & 1 & 3 & 34 \\
3 & 2 & 1 & 142 \\
3 & 2 & 2 & 1 \\
3 & 2 & 3 & 24 \\
3 & 3 & 1 & 317 \\
3 & 3 & 3 & 25 \\
\hline & & & \\
\hline
\end{tabular}

Man beachte, daß die Kombinationen A3 B1 C2 fehlen; hier liegen keine Beobachtungen vor; wir behandeln sie als strukturelle Nullen.

Da uns nur der Einfluß von $A$ und $B$ auf $C$ interessiert, nicht aber der Zusammenhang von $A$ und $B$, geben wir die Randverteilung von $A B$ als gegeben vor und passen zunächst das Modell 
$E y_{i j k}=\mu_{i j k}, \mu_{i j k}=\ln \mu_{i j k}$

(5.3) $\eta_{\mathrm{ijk}}=\beta_{\mathrm{o}}+\beta_{\mathrm{i}}^{\mathrm{A}}+\beta_{\mathrm{j}}^{\mathrm{B}}+\beta_{\mathrm{k}}^{\mathrm{C}}+\beta_{\mathrm{ij}}^{\mathrm{AB}}+\beta_{\mathrm{ik}}^{\mathrm{AC}}+$ $\beta_{\mathrm{jk}}^{\mathrm{BC}}, \mathrm{i}, \mathrm{j}, \mathrm{k}=1,2,3$

an.

Auf Grund der großen Stichprobe erhalten wir $D(c, f)=36.53$ mit $\mathrm{df}=6$,

so daß dieses Modell mit $\alpha=0.05$ verworfen werden muß. Wir berechnen daher die Parameter des saturierten Modells

(5.4) $\eta_{\mathrm{ijk}}=\beta_{\mathrm{o}}+\beta_{\mathrm{i}}^{\mathrm{A}}+\beta_{\mathrm{j}}^{\mathrm{B}}+\beta_{\mathrm{k}}^{\mathrm{C}}+\beta_{\mathrm{ij}}^{\mathrm{AB}}+\beta_{\mathrm{ik}}^{\mathrm{AC}}+$ $\beta_{j k}^{B C}+\beta_{i j k}^{A B C} i, j, k=1,2,3$

und erhalten bei Reparametrisierung durch corned effects bezogen auf die jeweils erste Kategorie:

TABELLE 11 Geschätzte Parameter des saturierten Modells

Error Poisson Link Log

Linear Predictor

GM A B C A.B A.C B.C A.B.C

\begin{tabular}{|c|c|c|c|}
\hline 1 & ESTIMATE & S.E & PARAMETER \\
\hline 1 & 6.385 & $.4107 \mathrm{E}-01$ & GM \\
\hline 2 & -.2156 & $.6147 \mathrm{E}-01$ & $A(2)$ \\
\hline 3 & -3.053 & .1934 & $A(3)$ \\
\hline 4 & 2.069 & $.435 \mathrm{OE}-01$ & B (2) \\
\hline 5 & 2.061 & $.4360 \mathrm{E}-0 \mathrm{I}$ & B (3) \\
\hline 6 & .2092 & $.5527 \mathrm{E}-01$ & $C(2)$ \\
\hline 7 & -1.220 & $.8603 E-01$ & $C(3)$ \\
\hline 8 & .1616 & $.6493 \mathrm{E}-01$ & $A(2) . B(2)$ \\
\hline 9 & .4163 & $.6457 \mathrm{E}-01$ & $A(2) \cdot B(3)$ \\
\hline 10 & -.4459 & .2213 & $A(3) . B(2)$ \\
\hline 11 & .3658 & .2019 & $A(3) \cdot B(3)$ \\
\hline 12 & -1.132 & .1020 & $A(2) . C(2)$ \\
\hline 13 & 2.136 & .1016 & $A(2) . C(3)$ \\
\hline 14 & -4.124 & 1.003 & $A(3) \cdot C(2)$ \\
\hline 15 & 1.415 & .2693 & $A(3), C(3)$ \\
\hline 16 & -1.041 & $.6129 \mathrm{E}-01$ & $B(2) \cdot C(2)$ \\
\hline 17 & -1.450 & .1034 & $\mathrm{~B}(2) \cdot \mathrm{C}(3)$ \\
\hline 18 & -2.086 & $.6835 \mathrm{E}-01$ & $B(3) \cdot C(2)$ \\
\hline 19 & -2.455 & .1268 & $B(3) \cdot C(3)$ \\
\hline 20 & $-.2551 \mathrm{E}-01$ & .1139 & $A(2) \cdot B(2) \cdot C(2)$ \\
\hline 21 & -.6773 & .1208 & $A(2) \cdot B(2) \cdot C(3)$ \\
\hline 22 & -.1290 & .1275 & $A(2) . B(3) . C(2)$ \\
\hline 23 & -.5448 & .1436 & $A(2) \cdot B(3) \cdot C(3)$ \\
\hline 24 & ZERO & ALIASED & $A(3) . B(2) . C(2)$ \\
\hline 25 & -.5215 & .3529 & $A(3) \cdot B(2) \cdot C(3)$ \\
\hline 26 & ZERO & ALIASED & $A(3) \cdot B(3) . C(2)$ \\
\hline 27 & -.2791 & .3527 & $A(3) . B(3) . C(3)$ \\
\hline
\end{tabular}

Auf Grund der fehlenden Beobachtungen wer$\operatorname{den} \beta_{322}^{\mathrm{ABC}}=\beta_{332}^{\mathrm{ABC}}=0$ gesetzt und als nicht schätzbar durch ALIASED ausgewiesen.

Zur Analyse der Wirkung von A,B auf $C$ betrachten wir, wie oben vorgeschlagen, zunächst das Verhältnis der Wahrscheinlichkeit, un/angelernte Arbeiterin zu sein, zur Wahrscheinlichkeit, nicht erwerbstätig zu sein.

$\eta_{\mathrm{ij} 2}-\eta_{\mathrm{ij} 1}=\beta_{2}^{\mathrm{C}}+\beta_{\mathrm{i} 2}^{\mathrm{AC}}+\beta_{\mathrm{j} 2}^{\mathrm{BC}}+\beta_{\mathrm{ij} 2}^{\mathrm{ABC}}$

$\beta_{2}^{C}=.2092$

$\beta_{22}^{\mathrm{AC}}=-1.132$

$\beta_{32}^{\mathrm{AC}}=-4.124$

$\beta_{22}^{\mathrm{BC}}=-1.041$

$\beta_{32}^{\mathrm{BC}}=-2.086$

Interpretiert man nun die Haupteffekte, wird die Wahrscheinlichkeit - im Vergleich zu Nichterwerbstätigen - an/ungelernte Arbeiterin zu sein, bei mittlerer bzw. höherer Ausbildung und bei Versorgung von Kindern wesentlich vermindert. Vergleicht man die Größe dieser Haupteffekte im logit-Modell mit den Interaktionseffekten, erkennt man sofort die relativ geringe Bedeutung der Interaktionswirkung von A.B auf C.

Ähnlich verhält es sich mit den logits, die das Verhältnis der Wahrscheinlichkeit, Angestellte oder Beamte zu sein, zur Wahrscheinlichkeit, nicht erwerbstätig zu sein, angeben.

$\eta_{\mathrm{ij} 3}-\eta_{\mathrm{ij} 1}=\beta_{3}^{\mathrm{C}}+\beta_{\mathrm{i} 3}^{\mathrm{AC}}+\beta_{\mathrm{j} 3}^{\mathrm{BC}}+\beta_{\mathrm{ij} 3}^{\mathrm{ABC}}$

$\beta_{3}^{C}=-.1220$

$\beta_{23}^{\mathrm{AC}}=2.136$

$\beta_{33}^{\mathrm{AC}}=1.415$

$\beta_{23}^{\mathrm{BC}}=-1.450$

$\beta_{33}^{\mathrm{BC}}=-2.455$

Wie zu erwarten war, wirkt $B$ in gleicher Weise auf $\mathrm{C} 3$ wie auf C2. Die Wahrscheinlichkeit der Berufstätigkeit vermindert sich, wenn Kinder zu versorgen sind.

Genau in die andere Richtung wirkt jedoch Variable A auf C3. Die Wahrscheinlichkeit, als An- 
gestellte oder Beamte tätig zu sein, wird bei mittlerer oder höherer Ausbildung beträchtlich erhöht.

\section{Weitere Anwendungen}

Neben den hier angegebenen Anwendungen des GLM-Ansatzes lassen sich auch folgende, in der sozialwissenschaftlichen Forschung mehrfach diskutierte Probleme und Verfahren mit GL-Modellen und geringfügigen Erweiterungen lösen:

- die Behandlung von ordinalen abhängigen Variablen,

- die Analyse von Verweildauern und Ubergangsraten bei inhomogenen Markoffprozessen, die in der Lebenszyklusforschung eine bedeutende Rolle spielen (Tuma et al. 1979), - die Maximum Likelihood-Schätzung von latent structure-Modellen (Goodman 1978).

\section{Literatur:}

Arminger, G., 1976: Loglineare Modelle zur Analyse nominal skalierter Variablen. Wien: Verband der wissenschaftlichen Gesellschaften Österreichs.

Arminger, G., 1979: Loglineare Weise zur Analyse des Zusammenhangs $z$ wischen nominalen Variablen. S. 218-261 in: K. Holm (Hrsg.), Die Befragung 6 . München: Francke.

Arminger, G., 1982: Klassische Anwendungen verallgemeinerter linearer Modelle in der empirischen Sozialforschung. S. 1-124 in: ZUMA - Arbeitsbericht Nr. 1982/03, Verallgemeinerte lineare Modelle in der empirischen Sozialforschung. NONMET/GLIM Workshop. Mannheim: ZUMA.

Birch, M.W., 1963: Maximum likelihood in three-way contingency tables. Journal of the Royal Statistical Society, Ser. B., 25: 220-233.

Bishop, Y.M.M./S.E. Fienberg/P.W. Holland, 1975: Discrete multivariate analysis: Theory and Practice. Cambridge: MIT Press.

Bock, R.D., 1975: Multivariate statistical methods in behaviorial research. New York: McGraw-Hill.

Cramer, J.C., 1980: Fertility and female employment: problems of causal direction. American Sociological Review 45: 167-190.

GLIM Manual, 1978: Oxford: Numerical Algorithm Group.

Goethe, J.W., von, 1796: Wilhelm Meisters Lehrjahre. Leipzig: Insel (1914).
Goodman, L.A., 1970: The multivariate analy sis of qualitative data: Interactions among multiple classifications. Journal of the American Statistical Association 65 : $226-256$.

Goodman, L.A., 1972: A general model for the analysis of surveys. American Journal of Sociology 77: $1035-1086$.

Goodman, L.A., 1978: Analyzing qualitative/categorial data: Log-linear models and latent structure analysis. Cambridge: Abt Books.

Grizzle, J.E./C.F. Starmer/G.G. Koch, 1969: Analysis of categorical data by linear models. Biometrics 25 : 489-504.

Haberman, S.H., 1974: The analysis of frequency data. Chicago: University of Chicago Press.

Handl, J., 1981 : Bildungsexpansion und berufliche Statuschancen von Frauen: Ein sozialhistorischer Versuch. Mannheim: Arbeitspapier Nr. 20 des VASMA Projekts an der Universität Mannheim.

Holm, K., 1979: Das allgemeine lineare Modell. S. 1-261 in: K. Holm (Hrsg.), Die Befragung 6 . München: Francke.

Küchler, M., 1978: Alternativen in der Kreuztabellenanalyse - Ein Vergleich zwischen Goodmans ,General Model" (ECTA) und dem Verfahren gewichteter Regression nach Grizzle et al. (NONMET II). Zeitschrift für Soziologie 7: 347-365.

Küchler, M., 1979: Multivariate Analyseverfahren. Stuttgart: Teubner.

Langeheine, R., 1979: Multivariate Analyse nominal skalierter Daten via Goodmans Modell: Sehr wohl eine Alternative. Zeitschrift für Soziologie 8: 380-390.

Langeheine, R., 1980: Log-lineare Modelle zur multivariaten Analyse qualitativer Daten. Eine Einführung. München: Oldenbourg.

Mincer, J., S. Polachek, 1974: Family investment investment in human capital: Earnings of women. Joumal of Political Economy 82: 76-108.

Müller, W., 1981: Familienzyklus und Frauenerwerbstätigkeit: Eine Analyse sozialen Wandels aus der Perspektive des Lebenslaufs. Mannheim: Arbeitspapier Nr. 21 des VASMA Projekts an der Universität Mannheim.

Nelder, J.A./R.W.M. Wedderburn, 1972: Generalized linear models, Journal of the Royal Statistical Society. Ser. A. 135 : 370-384.

Tuma, N.B./M.T. Hannan/L.P. Groeneveld, 1979: Dynamic Analysis of Event Histories in American Journal of Sociology 84: No. 4, 820-854.

Willms, A., 1981 : Integration auf Widerruf? Ein Beitrag zur Entwicklung des Verhältnisses von Frauenarbeit und Männerarbeit in Deutschland, 1882-1970. Mannheim: Arbeitspapier Nr. 22 des VASMA Projekts an der Universität Mannheim. 